\title{
XPS and SEM characterization for powder recycling within 3d printing process
}

\author{
Nima E. Gorji, Robert O'Connor and Dermot Brabazon
}

\author{
Nima E. Gorji. I-Form, Advanced Manufacturing Research Centre, Advanced Processing Technology Research Centre, School of \\ Mechanical and Manufacturing Engineering, Dublin City University, Dublin 9, Ireland. \\ Robert O'Connor. I-Form, Advanced Manufacturing Research Centre, Advanced Processing Technology Research Centre, School of \\ Mechanical and Manufacturing Engineering, Dublin City University, Dublin 9, Ireland. \\ Dermot Brabazon. I-Form, Advanced Manufacturing Research Centre, Advanced Processing Technology Research Centre, School \\ of Mechanical and Manufacturing Engineering, Dublin City University, Dublin 9, Ireland.
}

\begin{abstract}
In recent years, recycling the powder leftover within the additive manufacturing process has been attractive for both research, development and industry production. Powder recycling can significantly enhance the sustainability of the manufacturing process, reduce the cost and avoid producing metallic waste as a potential environmental hazard. The first step in reusing the recycled powders in the 3D printing process is to characterize the microstructure and surface quality of the powder for oxidation and impurity analysis. Here, scanning electron microscopy (SEM) and x-ray photoelectron spectroscopy (XPS) have been used for the morphology and surface composition analysis of the 316L powders within the Aconity 3D printer. A new powder collection strategy has been introduced to collect powders from different locations in the powder bed: from the top most and surface of the parts and powder bed after the print terminated, from between the printed parts at different heights. The XPS measurements revealed that oxidation is a common in all the powders compared to virgin powder and more oxidation was detected from the powders collected on the very top of the leftover powder and from surface of the bed. The size of the particles does not change much but larger particles remained at the topmost surface. This finding would help in designing a protocol for collecting the recycled powder from the powder bed and it is suggested to follow a a procedure of collecting powders from the different sections of the powder bed in order to avoid mixing the most and least affected particles.
\end{abstract}

Keywords. Powder Recycling, Additive Manufacturing, Surface Composition Spectroscopy, Microstructure Characterization, 3D Printing

\section{Introduction}

A large cost of Powder Bed Fusion (PBD) is associated to metallic powder alloys. To drive the Additive manufacturing (AM) towards a rather sustainable process, powder recycling must become more efficient and reliable during the 3D printing process $[1,2]$. This becomes more important by noting that the end-goal of AM is transition from prototyping to large scale production. Obviously, a vital aspect of this plan is to think of more economical and environment friendly AM process, e.g. by cutting the process cost and reducing the process waste [3,4]. Both goals can be achieved to a high extent by recycling the metallic powder in AM process. Reusing the feedstock powder in the PBF process has attracted the attention of researchers and technicians with the goal to reuse the powders as many times as possible without losing the mechanical properties of the printed parts. Currently, there are few approaches followed by the researchers on powder recycling: mixing the virgin powder with the recycled powder and sieving the recycled powder [5]. However, systematic studies on the recycled powders and parts have shown a significant impact on mechanical properties of the parts printed from recycled powders reused with simple post-treatments (mixing or sieving) process. More detailed studies are required to optimize the number of reusing times, mixing ratio, sieving and surface cleaning of the powder particles in order to print parts with comparable quality to the ones printed from feedstock powders [1]. Most recycling plans were developed for plastic filaments in 3D printing industry. However, there are few movements in industry to develop the metallic powder recycling for risk-tolerant applications. In 2017, Sigma Labs company agreed to develop 
in-situ monitoring combined with mechanical property characterization of the recycled powders within PBF together with the US Department of Commerce's National Institute of Standards and Technology (NIST). They have developed a PrintRite3D INSPECT® software hoping to understand how changes in the powder translate into build-chamber behavior and a finished 3D printed part. Therefore, comprehensive characterization of the recycled powders is essential to ensure the printed parts from these used powders keep a high quality. Microstructure and surface composition analyses are of the first vital characterization techniques required to estimate the size distribution and surface quality of the particles, level/thickness of oxidation or the presence of impurities or metallic oxides and agglomeration $[3,4]$. Scanning Electron Microscopy (SEM) and X-ray photoelectron spectroscopy (XPS) have been used here for powder recycling purposes. The XPS measurements are presented on 6 different powders sampled from the different areas of the powder bed. The aim is to compare the size distribution and surface quality of the recycled powders collected from across the powder bed. The conclusion will help to set a protocol for collecting the powder leftover in order to keep the least impacted powder from the rest which helps to print parts with a higher quality.

\section{Materials and Methods}

The Steel 316L powder was purchased from commercial producers LPW Carpenter additive and the powder average particle size are $45 \mu \mathrm{m}$ with apparent density of virgin powder of $7.98 \mathrm{~g} / \mathrm{cm}^{3}$. The recycled powders were sieved for $50 \mu \mathrm{m}$. We performed scanning electron microscopy (SEM) using EVO-LS15 Zeiss Scanning Electron Microscope to survey the size distribution, morphology and surface condition of the powders. The surface composition was also characterized by our VG Microtech electron spectrometer, at base pressure of $1^{\prime} 10^{-9}$ mbar, and a conventional Mg $\mathrm{K}_{\alpha}$ $(\mathrm{h} v=1253.6 \mathrm{eV}) \mathrm{X}$-ray source. CASAxps software has been used for data extraction from XPS spectra. The XPS spectra were calibrated to carbon peak of $285 \mathrm{eV}$ before further analysis. No chemical treatments applied before running XPS scans so to avoid surface contamination. The acquisition conditions were $20 \mathrm{eV}$ pass energy, at $0.1 \mathrm{eV}$ step $0.1 \mathrm{eV}$ and $30^{\circ}$ take-off angle. The Aconity 3D-printing machine was used to print several tensile cube parts and the reused powders were collected from its powder bed.

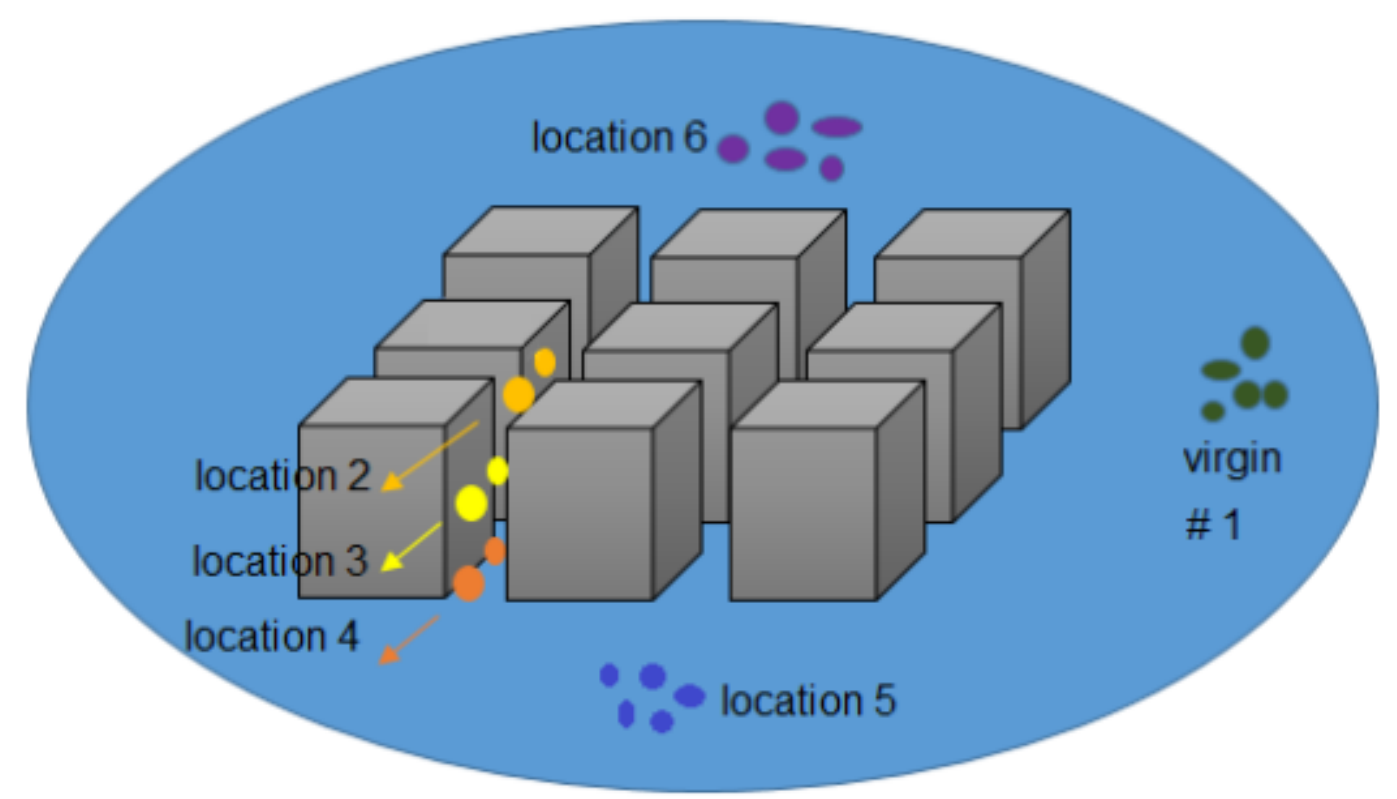

Fig. 1. Sampling locations on powder bed where the recycled powder was collected from between the cubes from top (\#2), middle (\#3) and bottom (\#4) of printed test cubes, powder bed surface (\#5) and from the overflow collector (\#6), all compared with virgin (\#1). 


\section{Results and Discussions}

The morphology and surface composition analysis of the virgin and the 5 recycled powders collected from different locations of the powder bed are presented in Fig. 2-5 after SEM and XPS characterizations. The powders were sampled from different locations of the powder bed as shown in Fig. 1. The first badge is virgin powder (\#1), the second badge was collected from between the test cubes from top (\#2) and the third (\#3) and fourth (\#4) badge collected from middle and bottom locations. The fifth badge (\#5) was collected from top surface of the powder bed after the built was over which is actually where the most powder leftover is collected. The sixth powder was sampled from overflow of the powders (\#6) on very top of the powders remained on the whole system.

\subsection{SEM measurements}

The SEM images (Fig. 2 and 3) show the microstructure of the virgin powder and the powders sampled from the different locations of the powder bed (\#5 and \#6). The powders collected from these locations shown to slightly more impacted during the AM process. Larger particles either with irregular shape non-spherical, unclean surface more satellites, bonded particles, coarse particles, and agglomeration is observed more in these powders compared to virgin powder with cleaner surface and more spherical shape. The size distribution is different by $<10 \%$ for the powders collected from these locations. We do see some spatters and open particles present within these powders which could pass through the sieving process. These results are in agreement with the SEM images presented in [6-9]. It is known that the particle size distribution and volume will increase by the number of build cycles which will, in turn, impact on the part density and mechanical properties. Comparing these images suggests that the powders remained on the most top and bottom sides of the powders bed must be collected in advance of the vacuuming out the leftover powder from the powder bed and if possible, to be kept in a separate recycling container without being mixed with other powders. On the other hand, the powders collected from between the printed cubes are mostly in regular circular shape with less satellites on the surface. However, these powders seem smaller in size. As shown in Fig. 3, the powders collected from location 2 and 4 are slightly more affected on the surface compared to powders of location 3 . This might be because location 3 is confined within the upper and bottom remained powders and the powders driven away from the laser irradiation are drifted to location 6 or remain on location 2 or fall on location 5 on the powder bed after the powder collector covers the surface with new powder. The ImageJ analysis of the powder images were performed to measure the average size of the particles for every location and compared them to the virgin powder as shown in Table 1. 

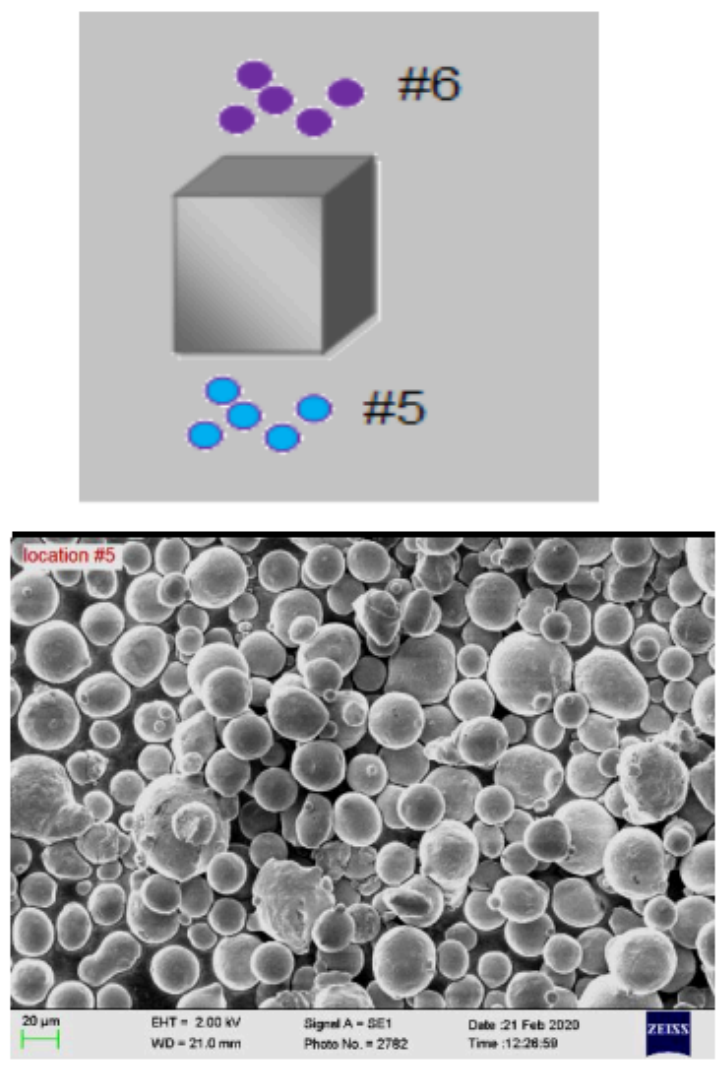

Fig. 2. SEM images taken from virgin (\#1) and the recycled powders with worst surface quality and size distribution changes collected from powder bed (\#5) and overflow collector (\#6).
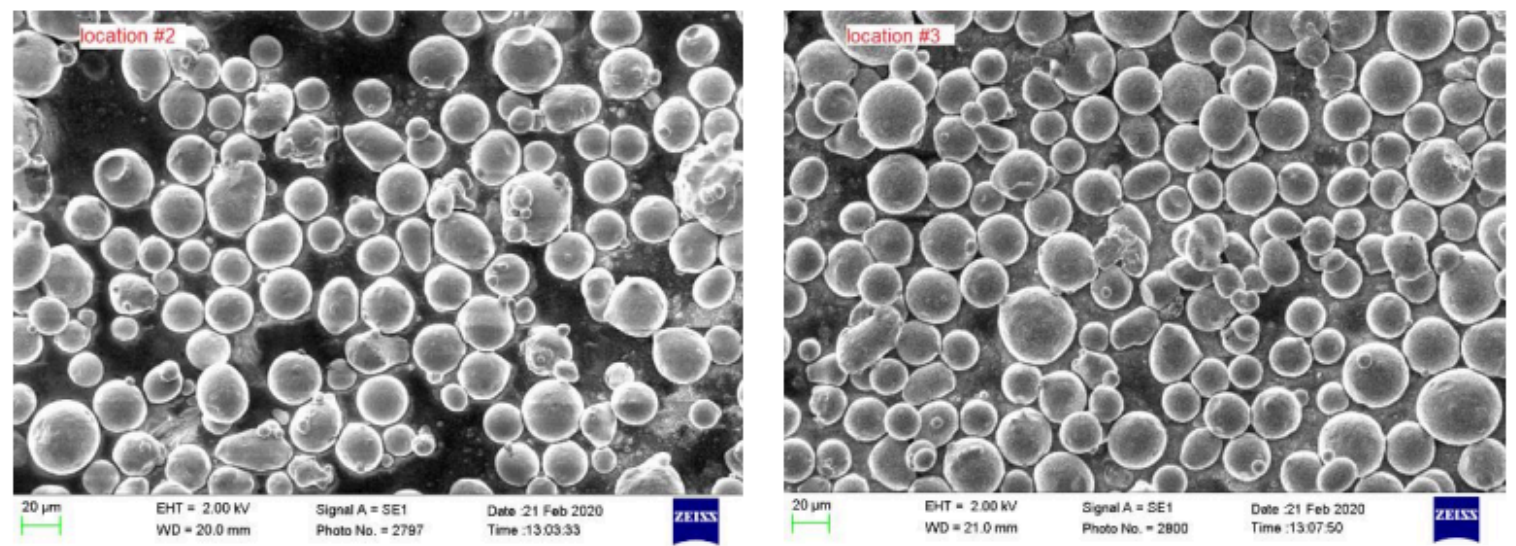

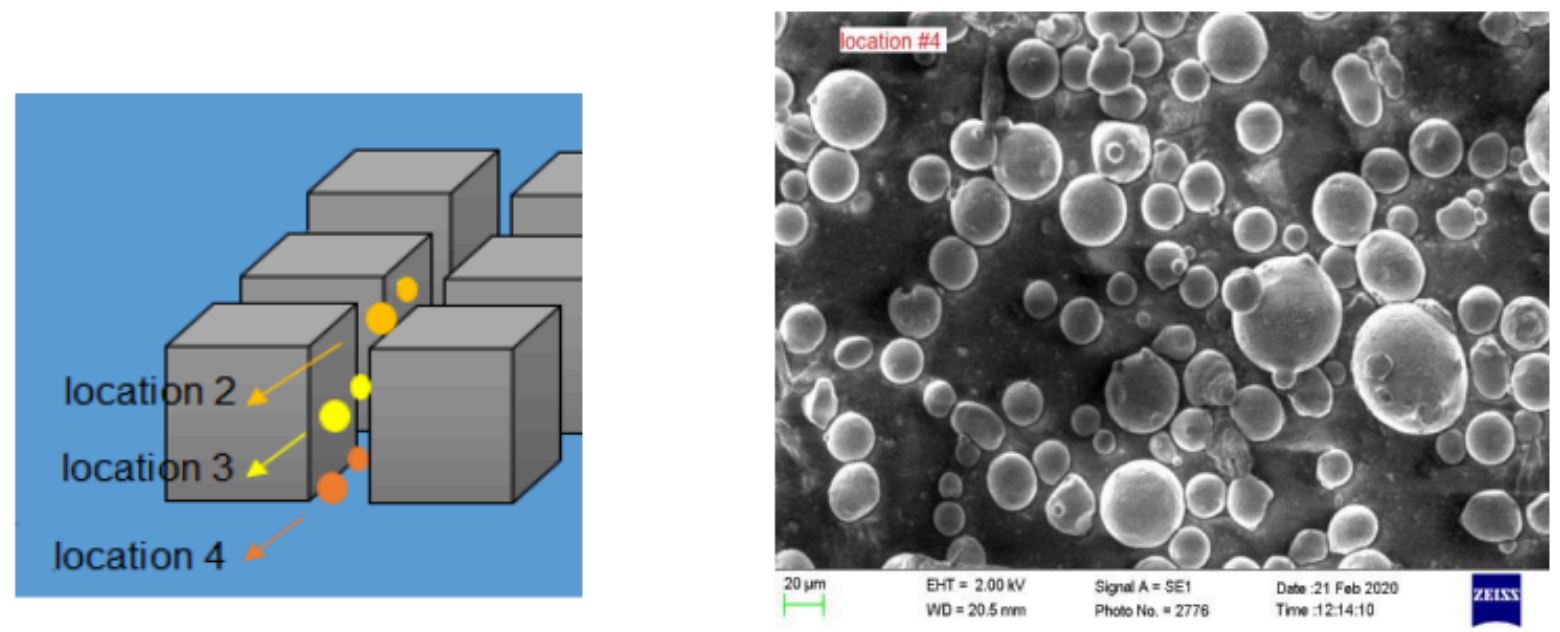

Fig. 3. SEM images taken from between the printed cubes from location \#2 at the top and between the cubes, location \#3 middle between and location \#4 bottom between the printed cubes.

Table 1. The average size of particles from each location as determined via SEM images processed using ImageJ software.

\begin{tabular}{|l|l|l|l|l|l|l|}
\hline Table 1 & Virgin & $\# 2$ & $\# 3$ & $\# 4$ & $\# 5$ & $\# 6$ \\
\hline Ave. size & 29.4 & 19.2 & 20.7 & 21.6 & 30 & 29 \\
\hline
\end{tabular}

The results as expected and mentioned above represent a comparable size of the virgin powder parties to the ones collected from location 5 and 6 whereas the particles from \#2, \#3, and \#4 were showing a similar range of sizes instead.

\subsection{XPS measurements}

In order to investigate the quality of the recycled powder for subsequent reuse cycles, we have performed XPS scanning of the powder particles. The XPS measurements presented in Fig. 4 for both virgin and recycled powders collected from different locations of the powder bed. Number of elements were detected on the surface such as Si, Oxygen, Mn, Fe and Carbon. The peaks are similar in general but analysis of the peaks gives slightly different concentration of elements on the surface $[1,7]$. 


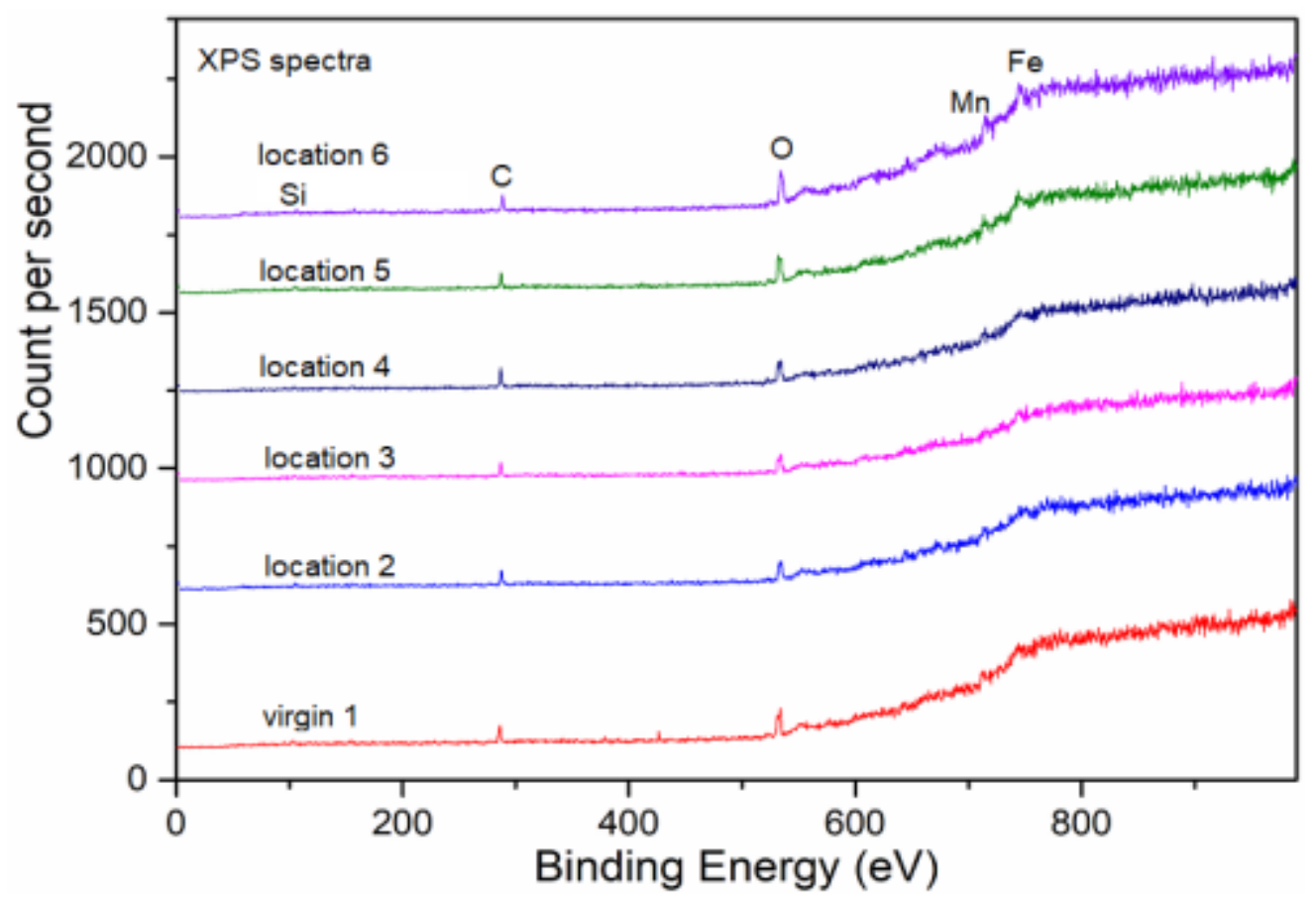

Fig. 4. XPS measurements on both virgin and recycled powders collected from different locations of the powder bed according the protocol shown in Figure 1.

Fig. 5 shows the Oxygen peaks of all the powders including the virgin powder. The Oxygen level on the powder surface has increased from $28.64 \%$ on the virgin powder to $40.6 \%$ and $36 \%$ on the powders collected from location \#5 and \#6. The Oxygen concentration was measured by CasaXPS software after analysis of the XPS spectra presented in Fig. 4. Caution might be noted for presenting such oxygen levels as the powders maybe affected from the moisture of the room and container during powder feeding, vacuuming and storing. However, the results indicate that the powders remained on the top of the parts and on the side bottom of the powder bed are more exposed to oxidation for a longer duration which is rational. The XPS spectra also shows a higher amount of oxygen on powders of location 2, 3, and 4 to about $29 \%, 30.3 \%$ and $29.5 \%$, respectively. The concentration of the other elements on the surface of powders collected from different locations has been summarized in Table 2. The concentration level of the Mn and Fe elements does not change much at different locations of the powder bed. The least level obtained from the location 3 which is confined between the printed parts and across the up and bottom of the parts (vertical direction). 


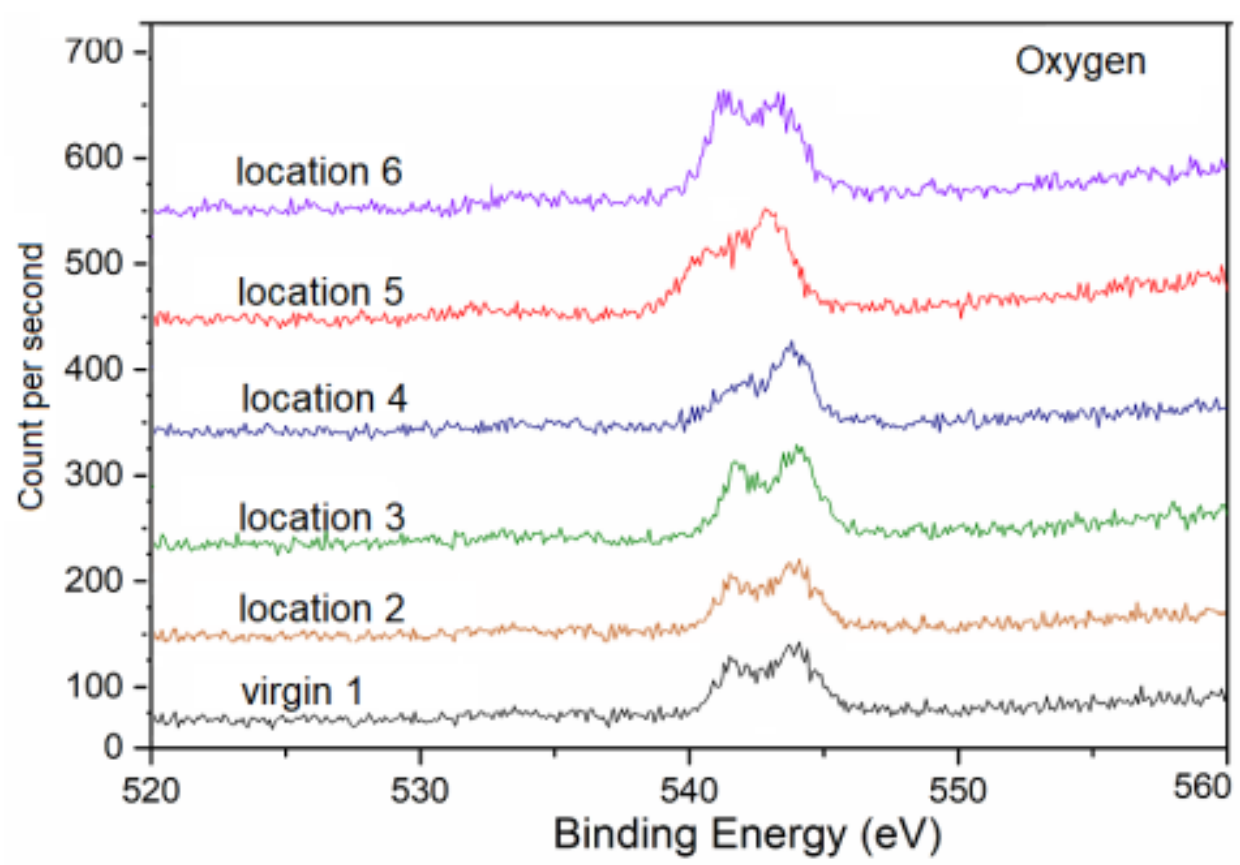

Fig. 5. Oxygen concentration measured by the XPS analysis on the surface of the recycled powders collected from the different locations of the powder bed.

The Mn value is almost the same compared to virgin powder and the Fe concentration does not either change on powders located in 5 and 6 but slightly lower in location 2 and 3. This means that other than the oxygen level increment in \#5 and \#6, the oxygen level is not increasing much on the other locations. The Si level is low in all areas but slightly higher in \#3 which is where the powder is more confined and the heat convection might be impeded and the Si diffuses out to the surface under a slightly higher temperature. These results are in agreement with the EDX results presented in Ref. $[3,4]$ where the change in concentration of these elements was reported to be negligible to about $\pm 2 \%$.

Table 2. Concentration of chemical elements on surface of virgin and recycled powders collected from different locations of the powder bed; the values were determined from the recorded XPS spectra.

\begin{tabular}{|l|c|c|c|c|c|}
\hline Table 2 & Oxygen & Carbon & Si & Mn & Fe \\
\hline Virgin & 28.64 & 32.6 & 5.3 & 14 & 19.3 \\
\hline Location 2 & 29 & 33.5 & 7 & 13 & 17.3 \\
\hline Location 3 & 30.3 & 33.7 & 8 & 12 & 16.5 \\
\hline Location 4 & 29.5 & 31 & 3.3 & 15 & 21 \\
\hline Location 5 & 40.6 & 36 & 6.3 & 14 & 18 \\
\hline Location 6 & 36 & 27 & 6.5 & 13 & 18.3 \\
\hline
\end{tabular}

\section{Conclusions}

The microstructure and surface composition of the metallic 316L powders has been investigated for recyclability analysis within the additive manufacture powder bed fusion process. The leftover powder has been collected from 5 different locations of the powder bed including from between the parts with different height and has been 
characterized using SEM and XPS techniques. The particle size distribution and concentration of metallic elements on these powders has been analysed and compared with the virgin counterpart. The most affected leftover powders were observed in location 5 (from powder bed) and 6 (overflow collector) after the print completed with 10 mm bigger size than the ones collected from between the parts. The oxygen concentration is also around $10 \%$ more on these powders 5 and 6. The powders remained between the parts (location 2, 3, and 4) are smaller in size (av. $20 \mathrm{~mm}$ ) and with lower oxygen concentration (av. $29 \%$ ) which might be due to confinement of this particles between the parts and the bottom/top powders spread by the coater. This research suggests that the powder collection from the 3D printer could follow a recycling protocol where the powders remaining on powder bed and on the overflow could be collected and stored separately from the powder collected from the between the parts. The research finding in this work can thereby allow for increased reusage and sustainability of powder consumption from the additive manufacturing process.

\section{Acknowledgment}

This research is supported by a research grant from Science Foundation Ireland (SFI) under Grant Number 16/RC/ 3872 and is co-funded under the European Regional Development Fund; and the New Foundation Award (No. P60687) received from Irish Research Council (2019) and also the ECIU Research Mobility (No. P60617) grant (2018).

\section{Bibliography}

[1] Ahmed F., Ali U., Sarker D., Marzbanrad E., Choi K., Mahmoodkhani Y., Toyserkani E., Study of powder recycling and its effect on printed parts during laser powder-bed fusion of 17-4 PH stainless steel, J. of Materials Processing Tech. J., 2020, 1, pp. 116-522. https://doi.org/10.1016/j.jmatprotec.2019.116522.

[2] Daraban A., Negrea C., Artimon F., Angelescu D., Popan G., Gheorghe S., Gheorghe M, Deep Look at Metal Additive Manufacturing Recycling and Use Tools for Sustainability Performance, Sustainability, 2019, 11:19, 5494, pp.1-20. https://doi.org/10.3390/su11195494.

[3] Gorji N. E., O'Connor R., Brabazon D., XPS, XRD, and SEM characterization of the virgin and recycled metallic powders for 3D printing applications, IOP Conference Series: Materials Science and Engineering, 2019, 591, 1012016. doi:10.1088/1757-899X/591/1/012016.

[4] Gorji, N.E., Saxena, P., Corfield, M., (...), Raghavendra, R., Brabazon, D., A new method for assessing the recyclability of powders within Powder Bed Fusion process, Materials Characterization, 2020, 161, 110167. https://doi.org/10.1016/ j.matchar.2020.110167

[5] Available from: https://3dprintingindustry.com/news/3d-printing-industry-review-of-the-yearapril-2019-166697/.

[6] Lutter-Günther M., Gebbe Ch., Kamps T., Seidel Ch., Reinhart G., Powder recycling in laser beam melting: strategies, consumption modeling and influence on resource efficiency, Production Engineering, 2018, 12, pp. 377-389. https://doi.org/10.1007/s11740-018-0790-7.

[7] Quinn P., O'Halloran S., Lawlor J., Raghavendra R., The effect of metal EOS 316L stainless steel additive manufacturing powder recycling on part characteristics and powder reusability, Advances in Materials and Processing Technologies, 2019, 1, pp. 1-3. https://doi.org/10.1080/2374068X.2019.1594602.

[8] Re F., Contaldi V., Astarita A., Palumbo B., Squillace A., Corrado P., Petta P., Statistical approach for assessing the effect 
of powder reuse on the final quality of AlSi10Mg parts produced by laser powder bed fusion additive manufacturing, The International Journal of Advanced Manufacturing Technology, 97, 2018, pp. 2231-2240. https://doi.org/10.1007/ s00170-018-2090-y.

[9] Sutton A., Kriewall C., Karnatia S., Leua M., Newkirk J., Characterization of AISI 304L stainless steel powder recycled in the laser powder-bed fusion process, Additive Manufacturing, 2020, 32, 100981. https://doi.org/10.1016/ j.addma.2019.100981.

[10] Vock S., Klöden B., Kirchner A., Weißgärber T., Kieback B., Powders for powder bed fusion: a review, Progress in Additive Manufacturing, 2019, 4, pp. 383-397. https://doi.org/10.1007/s40964-019-00078-6.

PDF automatically generated on 2021-05-20 06:26:09

Article url: https://popups.uliege.be/esaform21/index.php?id=403

published by ULiège Library in Open Access under the terms and conditions of the CC-BY License (https://creativecommons.org/licenses/by/4.0) 